\title{
A Fixed Point Approach to the Stability of an Additive-Quadratic-Cubic-Quartic Type Functional Equation
}

\author{
Yang-Hi Lee ${ }^{1}$ and Soon-Mo Jung ${ }^{2}$ \\ ${ }^{1}$ Department of Mathematics Education, Gongju National University of Education, Gongju 32553, Republic of Korea \\ ${ }^{2}$ Mathematics Section, College of Science and Technology, Hongik University, Sejong 30016, Republic of Korea \\ Correspondence should be addressed to Soon-Mo Jung; smjung@hongik.ac.kr
}

Received 4 December 2015; Accepted 17 March 2016

Academic Editor: Zbigniew Leśniak

Copyright ( $) 2016$ Y.-H. Lee and S.-M. Jung. This is an open access article distributed under the Creative Commons Attribution License, which permits unrestricted use, distribution, and reproduction in any medium, provided the original work is properly cited.

By applying a fixed point approach, we investigate the stability problems for an AQCQ functional equation of the form $f(x+2 y)+$ $f(x-2 y)-4 f(x+y)-4 f(x-y)+6 f(x)-f(2 y)-f(-2 y)+4 f(y)+4 f(-y)=0$.

\section{Introduction}

In 1940, Ulam [1] posed the problem concerning the stability of group homomorphisms. In the following year, Hyers [2] gave an affirmative answer to Ulam's problem for additive mappings between Banach spaces. Thereafter, many mathematicians came to deal with this subject (cf. $[3,4]$ ).

We now consider the following functional equation:

$$
\begin{aligned}
f(x & +2 y)+f(x-2 y)-4 f(x+y)-4 f(x-y) \\
& +6 f(x)-f(2 y)-f(-2 y)+4 f(y)+4 f(-y) \\
= & 0 .
\end{aligned}
$$

The mappings $f(x)=a x^{4}+b x^{3}+c x^{2}+d x$ are solutions of this functional equation, where $a, b, c, d$ are real constants.

The stability problems of (1) were investigated by Lee et al. [5]. Indeed, they proved the stability of the equation by dividing the relevant mapping into the additive, quadratic, cubic, and quartic parts and applying the fixed point method to each part separately. Unfortunately, they could not prove the uniqueness of the exact solution of (1) which approximates the (given) mapping that is a solution of the perturbed equation of (1).

In this paper, we will show that every solution of functional equation (1) is an AQCQ mapping (see below for the definition of AQCQ mapping) and we introduce a strictly contractive mapping which allows us to use the fixed point theory in the sense of Cădariu and Radu (see [6-8]). In a different way from Lee et al. [5], we do not split the (given) mapping which satisfies the perturbed equation of (1) and we apply the fixed point method to construct the explicit form of the exact solution of (1) near the (given) mapping by

$$
\begin{aligned}
F(x) & =\lim _{n \rightarrow \infty} \sum_{i=0}^{n}{ }_{n} C_{i}\left(\frac{(-1)^{n-i} 10^{i}}{16^{n}} f_{o}\left(2^{2 n-i} x\right)\right. \\
+ & \left.\frac{(-1)^{n-i} 20^{i}}{64^{n}} f_{e}\left(2^{2 n-i} x\right)\right)
\end{aligned}
$$

or

$$
\begin{aligned}
& F(x)=\lim _{n \rightarrow \infty} \sum_{i=0}^{n}{ }_{n} C_{i}\left(10^{i}(-16)^{n-i} f_{o}\left(\frac{x}{2^{2 n-i}}\right)\right. \\
& \left.+20^{i}(-64)^{n-i} f_{e}\left(\frac{x}{2^{2 n-i}}\right)\right),
\end{aligned}
$$

where we denote by $f_{o}$ and $f_{e}$ the odd and even parts of $f$, respectively.

Using this method, we can prove the uniqueness of the exact solution of (1) near the (given) mapping.

Throughout this paper, let $\mathbb{N}$ denote the set of all positive integers and let $\mathbb{N}_{0}$ denote the set of all nonnegative integers. 


\section{Preliminaries}

We recall the fixed point theorem of Diaz and Margolis [9].

Theorem 1. Assume that $(X, d)$ is a complete generalized metric space and $J: X \rightarrow X$ is a strictly contractive mapping with the Lipschitz constant $0<L<1$. It then holds that, for each $x \in X$, either $d\left(J^{n} x, J^{n+1} x\right)=\infty$ for all $n \in \mathbb{N}_{0}$ or there exists $k \in \mathbb{N}_{0}$ such that

(i) $d\left(J^{n} x, J^{n+1} x\right)<\infty$ for all $n \geq k$;

(ii) the sequence $\left\{J^{n} x\right\}$ is convergent to a fixed point $y^{*}$ of $J$;

(iii) $y^{*}$ is the unique fixed point of $J$ in $Y=\left\{y \in X: d\left(J^{k} x\right.\right.$, $y)<\infty\}$;

(iv) $d\left(y, y^{*}\right) \leq(1 /(1-L)) d(y, J y)$ for all $y \in Y$.

Throughout this paper, let $V$ and $W$ be real vector spaces, let $X$ be a real normed space, and let $Y$ be a real Banach space.

For a given mapping $f: V \rightarrow W$, we use the following abbreviations:

$$
\begin{aligned}
& f_{o}(x):= \frac{f(x)-f(-x)}{2}, \\
& f_{e}(x):= \frac{f(x)+f(-x)}{2}, \\
& A f(x, y):= f(x+y)-f(x)-f(y), \\
& Q f(x, y):= f(x+y)+f(x-y)-2 f(x) \\
&-2 f(y), \\
& C f(x, y):= f(x+2 y)-3 f(x+y)+3 f(x) \\
&-f(x-y)-6 f(y), \\
& Q^{\prime} f(x, y):= f(x+2 y)-4 f(x+y)+6 f(x) \\
&-4 f(x-y)+f(x-2 y)-24 f(y), \\
& E f(x, y):= f(x+2 y)-4 f(x+y)+6 f(x) \\
&-4 f(x-y)+f(x-2 y), \\
&+4 f(-y) \\
& D f(x, y):= f(x+2 y)+f(x-2 y)-4 f(x+y) \\
&-4 f(x-y)+6 f(x)-f(2 y) \\
&-f(-2 y)+4 f(y)+4 f(-y) \\
& E f(x, y)-f(2 y)-f(-2 y)+4 f(y) \\
& \hline f(x, y)-f(2 y)-f(-2 y)+28 f(y) \\
&=
\end{aligned}
$$

for all $x, y \in V$. Each solution of $A f(x, y)=0, Q f(x, y)=0$, $C f(x, y)=0$, and $Q^{\prime} f(x, y)=0$ is called an additive mapping, a quadratic mapping, a cubic mapping, and a quartic mapping, respectively. Every mapping $f: V \rightarrow W$ is called an AQCQ mapping (additive-quadratic-cubic-quartic mapping) if $f$ can be expressed by the sum of an additive mapping, a quadratic mapping, a cubic mapping, and a quartic mapping.

The following lemmas have been proved in [10].

Lemma 2 ([10], Theorem 2.3). If $f: V \rightarrow W$ is an odd mapping satisfying $E f(x, y)=0$ for all $x, y \in V$, then there exists a cubic mapping $C: V \rightarrow W$ and an additive mapping $A: V \rightarrow$ $W$ such that $f(x)=C(x)+A(x)$ for all $x \in V$.

Lemma 3 ([10], Theorem 2.4). If $f: V \rightarrow W$ is an even mapping satisfying the equalities $E f(x, y)=0$ for all $x, y \in V$ and $f(2 x)=4 f(x)$ for all $x \in V$, then $f$ is a quadratic mapping.

In the following theorem, we investigate the general solution of the functional equation $\operatorname{Df}(x, y)=0$.

Theorem 4. A mapping $f: V \rightarrow W$ satisfies $D f(x, y)=0$ for all $x, y \in V$ if and only if $f$ is an AQCQ mapping.

Proof. First, we assume that a mapping $f: V \rightarrow W$ satisfies $D f(x, y)=0$ for all $x, y \in V$. Then, the odd part $f_{o}$ satisfies the equalities

$$
\begin{aligned}
E f_{o}(x, y) & =D f_{o}(x, y)=\frac{1}{2} D f(x, y)-\frac{1}{2} D f(-x,-y) \\
& =0
\end{aligned}
$$

for all $x, y \in V$. It follows from Lemma 2 that $f_{o}$ is a cubicadditive mapping.

Let $f_{1}(x):=f_{e}(2 x)-16 f_{e}(x)$ and $f_{2}(x):=f_{e}(2 x)-$ $4 f_{e}(x)$ for all $x \in V$. By a direct calculation and using the assumption that $D f(x, y)=0$ for all $x, y \in V$, we obtain $f(0)=(1 / 6) D f(0,0)=0$ and

$$
\begin{aligned}
f_{1}(2 x)-4 f_{1}(x) & =f_{e}(4 x)-20 f_{e}(2 x)+64 f_{e}(x) \\
& =f_{2}(2 x)-16 f_{2}(x) \\
& =D f_{e}(2 x, x)+4 D f_{e}(x, x)=0 ;
\end{aligned}
$$

that is,

$$
\begin{aligned}
& f_{1}(2 x)=4 f_{1}(x), \\
& f_{2}(2 x)=16 f_{2}(x)
\end{aligned}
$$

for all $x \in V$. Using these equalities, we further get the equalities $E f_{1}(x, y)=D f_{1}(x, y)$ and $Q^{\prime} f_{2}(x, y)=D f_{2}(x, y)$ which imply the equalities $E f_{1}(x, y)=0$ and $Q^{\prime} f_{2}(x, y)=0$ for all $x, y \in V$. Hence, by (7) and the fact that $E f_{1}(x, y)=0$, Lemma 3 implies that $f_{1}$ is quadratic. Moreover, $f_{2}$ is quartic because of $Q^{\prime} f_{2}(x, y)=0$ for all $x, y \in V$. Since

$$
f(x)=f_{o}(x)+f_{e}(x)=f_{o}(x)-\frac{1}{12} f_{1}(x)+\frac{1}{12} f_{2}(x)
$$

for all $x \in V, f$ is an AQCQ mapping.

Conversely, assume that $f_{1}, f_{2}, f_{3}, f_{4}$ are mappings such that the equalities $f(x):=f_{1}(x)+f_{2}(x)+f_{3}(x)+f_{4}(x)$, 
$A f_{1}(x, y)=0, Q f_{2}(x, y)=0, C f_{3}(x, y)=0$, and $Q^{\prime} f_{4}(x, y)=$ 0 hold for all $x, y \in V$. Then the equalities $f_{1}(x)=-f_{1}(-x)$, $f_{2}(x)=f_{2}(-x), f_{3}(x)=-f_{3}(-x), f_{4}(x)=f_{4}(-x), f_{1}(2 x)=$ $2 f_{1}(x), f_{2}(2 x)=4 f_{2}(x), f_{3}(2 x)=8 f_{3}(x)$, and $f_{4}(2 x)=$ $16 f_{4}(x)$ hold for all $x \in V$. From the above equalities, we obtain the equalities

$$
\begin{aligned}
D f_{1}(x, y)= & 4 A f_{1}(x+y, x-y) \\
& -A f_{1}(x+2 y, x-2 y), \\
D f_{2}(x, y)= & Q f_{2}(x, 2 y)-4 Q f_{2}(x, y), \\
D f_{3}(x, y)= & C f_{3}(x, y)-C f_{3}(x-y, y), \\
D f_{4}(x, y)= & Q^{\prime} f_{4}(x, y)
\end{aligned}
$$

for all $x, y \in V$, which imply that

$$
\begin{aligned}
D f(x, y)= & D f_{1}(x, y)+D f_{2}(x, y)+D f_{3}(x, y) \\
& +D f_{4}(x, y)=0
\end{aligned}
$$

for all $x, y \in V$.

\section{Main Results}

Throughout this section, let $V$ be a real vector space and let $Y$ be a real Banach space.

In the following theorems, we can prove the generalized Hyers-Ulam stability of the functional equation (1) by using a fixed point approach.

Theorem 5. Let $f: V \rightarrow Y$ be a mapping, for which there exists a mapping $\varphi: V^{2} \rightarrow[0, \infty)$ such that the inequality

$$
\|D f(x, y)\| \leq \varphi(x, y)
$$

holds for all $x, y \in V$, and let $f(0)=0$. If there exists a constant $0<L<1$ such that $\varphi$ satisfies the condition

$$
\varphi(2 x, 2 y) \leq(\sqrt{41}-5) L \varphi(x, y)
$$

for all $x, y \in V$, then there exists a unique solution $F: V \rightarrow Y$ of (1) satisfying the inequality

$$
\|f(x)-F(x)\| \leq \frac{5}{128(1-L)} \Phi(x)
$$

for all $x \in V$, where $\Phi(x):=\varphi(2 x, x)+4 \varphi(x, x)+\varphi(-2 x,-x)+$ $4 \varphi(-x,-x)$. In particular, $F$ is represented by

$$
\begin{gathered}
F(x)=\lim _{n \rightarrow \infty} \sum_{i=0}^{n}{ }_{n} C_{i}\left(\frac{(-1)^{n-i} 10^{i}}{16^{n}} f_{o}\left(2^{2 n-i} x\right)\right. \\
\left.+\frac{(-1)^{n-i} 20^{i}}{64^{n}} f_{e}\left(2^{2 n-i} x\right)\right)
\end{gathered}
$$

for all $x \in V$.
Proof. Let $S$ be the set of all functions $g: V \rightarrow Y$ with $g(0)=$ 0 . We introduce a generalized metric on $S$ by

$$
\begin{aligned}
& d(g, h)=\inf \{K \in[0, \infty]:\|g(x)-h(x)\| \\
& \quad \leq K \Phi(x) \forall x \in V\} .
\end{aligned}
$$

It is not difficult to see that $(S, d)$ is a generalized complete metric space (see [11, 12]).

We now consider the mapping $J: S \rightarrow S$, which is defined by

$$
\begin{aligned}
J g(x):= & -\frac{5}{128} g(4 x)+\frac{3}{128} g(-4 x)+\frac{60}{128} g(2 x) \\
& -\frac{20}{128} g(-2 x)
\end{aligned}
$$

for all $x \in V$. We assert that the equality

$$
\begin{aligned}
J^{n} g(x)= & \sum_{i=0}^{n}{ }_{n} C_{i} \frac{(-1)^{n-i} 10^{i}}{16^{n}} g_{o}\left(2^{2 n-i} x\right) \\
& +\sum_{i=0}^{n}{ }_{n} C_{i} \frac{(-1)^{n-i} 20^{i}}{64^{n}} g_{e}\left(2^{2 n-i} x\right)
\end{aligned}
$$

holds for all $n \in \mathbb{N}_{0}$ and $x \in V$. Equality (17) is true if $n=0$. We assume that (17) is true for some $n \in \mathbb{N}_{0}$. Then we have

$$
\begin{aligned}
J^{n+1} g(x)= & J^{n}(J g(x)) \\
= & \sum_{i=0}^{n}{ }_{n} C_{i} \frac{(-1)^{n-i} 10^{i}}{16^{n}} J g_{o}\left(2^{2 n-i} x\right) \\
& +\sum_{i=0}^{n}{ }_{n} C_{i} \frac{(-1)^{n-i} 20^{i}}{64^{n}} J g_{e}\left(2^{2 n-i} x\right) \\
= & \sum_{i=0}^{n}{ }_{n} C_{i} \frac{(-1)^{n-i} 10^{i}}{16^{n}} \frac{-5}{128} g_{o}\left(2^{2 n+2-i} x\right) \\
& +\sum_{i=0}^{n}{ }_{n} C_{i} \frac{(-1)^{n-i} 10^{i}}{16^{n}} \frac{3}{128} g_{o}\left(-2^{2 n+2-i} x\right) \\
& +\sum_{i=0}^{n}{ }_{n} C_{i} \frac{(-1)^{n-i} 10^{i}}{16^{n}} \frac{60}{128} g_{o}\left(2^{2 n+1-i} x\right) \\
& +\sum_{i=0}^{n}{ }_{n} C_{i} \frac{(-1)^{n-i} 10^{i}}{16^{n}} \frac{-20}{128} g_{o}\left(-2^{2 n+1-i} x\right) \\
& +\sum_{i=0}^{n}{ }_{n} C_{i} \frac{(-1)^{n-i} 20^{i}}{64^{n}} \frac{-5}{128} g_{e}\left(2^{2 n+2-i} x\right) \\
& +\sum_{i=0}^{n}{ }_{n} C_{i} \frac{(-1)^{n-i} 20^{i}}{64^{n}} \frac{3}{128} g_{e}\left(-2^{2 n+2-i} x\right) \\
& +\sum_{i=0}^{n}{ }_{n} C_{i} \frac{(-1)^{n-i} 20^{i}}{64^{n}} \frac{60}{128} g_{e}\left(2^{2 n+1-i} x\right) \\
& +\sum_{i=0}^{n}{ }_{n} C_{i} \frac{(-1)^{n-i} 20^{i}}{64^{n}} \frac{-20}{128} g_{e}\left(-2^{2 n+1-i} x\right)
\end{aligned}
$$




$$
\begin{aligned}
= & \sum_{i=0}^{n}{ }_{n} C_{i} \frac{(-1)^{n+1-i} 10^{i}}{16^{n+1}} g_{o}\left(2^{2 n+2-i} x\right) \\
& +\sum_{i=0}^{n}{ }_{n} C_{i} \frac{(-1)^{n-i} 10^{i+1}}{16^{n+1}} g_{o}\left(2^{2 n+1-i} x\right) \\
& +\sum_{i=0}^{n}{ }_{n} C_{i} \frac{(-1)^{n+1-i} 20^{i}}{64^{n+1}} g_{e}\left(2^{2 n+2-i} x\right) \\
& +\sum_{i=0}^{n}{ }_{n} C_{i} \frac{(-1)^{n-i} 20^{i+1}}{64^{n+1}} g_{e}\left(2^{2 n+1-i} x\right) \\
= & \sum_{i=0}^{n+1}{ }_{n+1} C_{i} \frac{(-1)^{n+1-i} 10^{i}}{16^{n+1}} g_{o}\left(2^{2 n+2-i} x\right) \\
& +\sum_{i=0}^{n+1}{ }_{n+1} C_{i} \frac{(-1)^{n+1-i} 20^{i}}{64^{n+1}} g_{e}\left(2^{2 n+2-i} x\right)
\end{aligned}
$$

for all $n \in \mathbb{N}_{0}$ and $x \in V$, which we can obtain from (17) provided we replace $n$ with $n+1$.

Let $g, h \in S$ and let $K \in[0, \infty]$ be an arbitrary constant with $d(g, h) \leq K$. From the definition of $d$, we have

$$
\begin{aligned}
& \|J g(x)-J h(x)\| \leq \frac{5}{128}\|g(4 x)-h(4 x)\| \\
& +\frac{3}{128}\|g(-4 x)-h(-4 x)\|+\frac{60}{128} \| g(2 x)
\end{aligned}
$$

$$
\begin{aligned}
& -h(2 x)\left\|+\frac{20}{128}\right\| g(-2 x)-h(-2 x) \| \\
& \leq K\left(\frac{5}{128} \Phi(4 x)+\frac{3}{128} \Phi(-4 x)+\frac{60}{128} \Phi(2 x)\right. \\
& \left.+\frac{20}{128} \Phi(-2 x)\right) \leq K\left(\frac{8}{128} \Phi(4 x)+\frac{80}{128} \Phi(2 x)\right) \\
& \leq K\left(\frac{\sqrt{41}-5}{16} L \Phi(2 x)+\frac{10}{16} \Phi(2 x)\right) \\
& \leq K\left(\frac{(\sqrt{41}-5)^{2}}{16} L^{2} \Phi(x)\right. \\
& \left.+\frac{10(\sqrt{41}-5)}{16} L \Phi(x)\right) \leq K \\
& . \frac{(\sqrt{41}-5)^{2}+10(\sqrt{41}-5)}{16} L \Phi(x) \leq L K \Phi(x)
\end{aligned}
$$

for all $x \in V$, which implies that

$$
d(J g, J h) \leq L d(g, h)
$$

for any $g, h \in S$. That is, $J$ is a strictly contractive self-mapping of $S$ with the Lipschitz constant $L$.

Moreover, by a long calculation and (11), we see that

$$
\begin{aligned}
\|f(x)-J f(x)\| & =\frac{\|5 D f(2 x, x)+20 D f(x, x)-3 D f(-2 x,-x)-12 D f(-x,-x)\|}{128} \\
& \leq \frac{5 \varphi(2 x, x)+20 \varphi(x, x)+3 \varphi(-2 x,-x)+12 \varphi(-x,-x)}{128} \leq \frac{5}{128} \Phi(x)
\end{aligned}
$$

for all $x \in V$. It means that $d(f, J f) \leq 5 / 128<\infty$ by the definition of $d$. Therefore, according to Theorem 1(ii) and (iii), the sequence $\left\{J^{n} f\right\}$ converges to the unique fixed point $F: V \rightarrow Y$ of $J$ in the set $T=\{g \in S: d(f, g)<\infty\}$, which is represented by (14) for all $x \in V$.

Notice that

$$
d(f, F) \leq \frac{1}{1-L} d(f, J f) \leq \frac{5}{128(1-L)},
$$

which implies (13).

By the definition of $F$, together with (11) and (12), we have

$$
\begin{aligned}
& \|D F(x, y)\|=\lim _{n \rightarrow \infty}\left\|D J^{n} f(x, y)\right\| \\
& =\lim _{n \rightarrow \infty} \| \sum_{i=0}^{n}{ }_{n} C_{i} \frac{(-1)^{n-i} 10^{i}}{16^{n}} D f_{o}\left(2^{2 n-i} x, 2^{2 n-i} y\right) \\
& +\sum_{i=0}^{n}{ }_{n} C_{i} \frac{(-1)^{n-i} 20^{i}}{64^{n}} D f_{e}\left(2^{2 n-i} x, 2^{2 n-i} y\right) \|
\end{aligned}
$$

$$
\begin{aligned}
& \leq \lim _{n \rightarrow \infty} \sum_{i=0}^{n} \frac{{ }_{n} C_{i}}{2}\left(\frac{10^{i}}{16^{n}}+\frac{20^{i}}{64^{n}}\right) \times\left(\varphi\left(2^{2 n-i} x, 2^{2 n-i} y\right)\right. \\
& \left.+\varphi\left(-2^{2 n-i} x,-2^{2 n-i} y\right)\right) \leq \lim _{n \rightarrow \infty} \sum_{i=0}^{n}{ }_{n} C_{i} \frac{10^{i}}{16^{n}} \times(\sqrt{41} \\
& -5)^{n-i} L^{n-i}\left(\varphi\left(2^{n} x, 2^{n} y\right)+\varphi\left(-2^{n} x,-2^{n} y\right)\right) \\
& \leq \lim _{n \rightarrow \infty} \sum_{i=0}^{n}{ }_{n} C_{i} \frac{(\sqrt{41}-5)^{n-i} 10^{i}}{16^{n}}\left(\varphi\left(2^{n} x, 2^{n} y\right)\right. \\
& \left.+\varphi\left(-2^{n} x,-2^{n} y\right)\right) \\
& \leq \lim _{n \rightarrow \infty} \frac{(\sqrt{41}+5)^{n}}{16^{n}}\left(\varphi\left(2^{n} x, 2^{n} y\right)+\varphi\left(-2^{n} x\right.\right. \\
& \left.\left.-2^{n} y\right)\right) \leq \lim _{n \rightarrow \infty} \frac{(\sqrt{41}+5)^{n}(\sqrt{41}-5)^{n}}{16^{n}}
\end{aligned}
$$




$$
\begin{aligned}
& \cdot L^{n}(\varphi(x, y)+\varphi(-x,-y)) \leq \lim _{n \rightarrow \infty} L^{n}(\varphi(x, y) \\
& +\varphi(-x,-y))=0
\end{aligned}
$$

for all $x, y \in V$; that is, $F$ is a solution of the functional equation (1). Notice that if $F$ is a solution of the functional equation (1), then the equality

$$
\begin{aligned}
F(x)-J F(x)= & \frac{5}{128} D F(2 x, x)+\frac{20}{128} D F(x, x) \\
& -\frac{3}{128} D F(-2 x,-x) \\
& -\frac{12}{128} D F(-x,-x)
\end{aligned}
$$

implies that $F$ is a fixed point of $J$.

In the following theorem, we replace condition (12) for $\varphi$ with another (25) and prove the generalized Hyers-Ulam stability of AQCQ equation (1) by applying a fixed point approach.

Theorem 6. Let $f: V \rightarrow Y$ be a mapping, for which there exists a mapping $\varphi: V^{2} \rightarrow[0, \infty)$ such that inequality (11) holds for all $x, y \in V$, and let $f(0)=0$. If there exists a constant $0<L<1$ such that $\varphi$ satisfies the condition

$$
L \varphi(2 x, 2 y) \geq \frac{32}{\sqrt{41}-5} \varphi(x, y)
$$

for all $x, y \in V$, then there exists a unique solution $F: V \rightarrow Y$ of (1) satisfying the inequality

$$
\|f(x)-F(x)\| \leq \frac{(\sqrt{41}-5)^{2} L^{2}}{32^{2}(1-L)} \Phi(x)
$$

for all $x \in V$. In particular, $F$ is represented by

$$
\begin{aligned}
F(x) & =\lim _{n \rightarrow \infty} \sum_{i=0}^{n}{ }_{n} C_{i}\left(10^{i}(-16)^{n-i} f_{o}\left(\frac{x}{2^{2 n-i}}\right)\right. \\
& \left.+20^{i}(-64)^{n-i} f_{e}\left(\frac{x}{2^{2 n-i}}\right)\right)
\end{aligned}
$$

for all $x \in V$.

Proof. Let $(S, d)$ be the set defined in the proof of Theorem 5 . We now consider the mapping $J: S \rightarrow S$ defined by

$$
\begin{aligned}
J g(x):= & 15 g\left(\frac{x}{2}\right)+5 g\left(\frac{-x}{2}\right)-40 g\left(\frac{x}{4}\right) \\
& -24 g\left(\frac{-x}{4}\right)
\end{aligned}
$$

for all $x \in V$. We assert that the equality

$$
\begin{aligned}
J^{n} g(x)= & \sum_{i=0}^{n}{ }_{n} C_{i} 10^{i}(-16)^{n-i} g_{o}\left(\frac{x}{2^{2 n-i}}\right) \\
& +\sum_{i=0}^{n}{ }_{n} C_{i} 20^{i}(-64)^{n-i} g_{e}\left(\frac{x}{2^{2 n-i}}\right)
\end{aligned}
$$

holds for all $n \in \mathbb{N}$ and $x \in V$. Equality (29) is obviously true when $n=0$. We assume that (29) holds for some $n \in \mathbb{N}_{0}$. Then we have

$$
\begin{aligned}
& J^{n+1} g(x)=J^{n}(J g(x)) \\
& =\sum_{i=0}^{n}{ }_{n} C_{i} 10^{i}(-16)^{n-i} J g_{o}\left(\frac{x}{2^{2 n-i}}\right) \\
& +\sum_{i=0}^{n}{ }_{n} C_{i} 20^{i}(-64)^{n-i} J g_{e}\left(\frac{x}{2^{2 n-i}}\right) \\
& =\sum_{i=0}^{n}{ }_{n} C_{i} 10^{i}(-16)^{n-i} 15 g_{o}\left(\frac{x}{2^{2 n-1-i}}\right) \\
& +\sum_{i=0}^{n}{ }_{n} C_{i} 10^{i}(-16)^{n-i} 5 g_{o}\left(\frac{-x}{2^{2 n-1-i}}\right) \\
& -\sum_{i=0}^{n}{ }_{n} C_{i} 10^{i}(-16)^{n-i} 40 g_{o}\left(\frac{x}{2^{2 n-2-i}}\right) \\
& -\sum_{i=0}^{n}{ }_{n} C_{i} 10^{i}(-16)^{n-i} 24 g_{o}\left(\frac{-x}{2^{2 n-2-i}}\right) \\
& +\sum_{i=0}^{n}{ }_{n} C_{i} 20^{i}(-64)^{n-i} 15 g_{e}\left(\frac{x}{2^{2 n-1-i}}\right) \\
& +\sum_{i=0}^{n}{ }_{n} C_{i} 20^{i}(-64)^{n-i} 5 g_{e}\left(\frac{-x}{2^{2 n-1-i}}\right) \\
& -\sum_{i=0}^{n}{ }_{n} C_{i} 20^{i}(-64)^{n-i} 40 g_{e}\left(\frac{x}{2^{2 n-2-i}}\right) \\
& -\sum_{i=0}^{n}{ }_{n} C_{i} 20^{i}(-64)^{n-i} 24 g_{e}\left(\frac{-x}{2^{2 n-2-i}}\right) \\
& =\sum_{i=0}^{n}{ }_{n} C_{i} 10^{i+1}(-16)^{n-i} g_{o}\left(\frac{x}{2^{2 n-1-i}}\right) \\
& +\sum_{i=0}^{n}{ }_{n} C_{i} 10^{i}(-16)^{n+1-i} g_{o}\left(\frac{x}{2^{2 n-2-i}}\right) \\
& +\sum_{i=0}^{n}{ }_{n} C_{i} 20^{i+1}(-64)^{n-i} g_{e}\left(\frac{x}{2^{2 n-1-i}}\right) \\
& +\sum_{i=0}^{n}{ }_{n} C_{i} 20^{i}(-64)^{n+1-i} g_{e}\left(\frac{x}{2^{2 n-2-i}}\right) \\
& =\sum_{i=0}^{n+1}{ }_{n+1} C_{i} 10^{i}(-16)^{n+1-i} g_{o}\left(\frac{x}{2^{2 n+2-i}}\right) \\
& +\sum_{i=0}^{n+1}{ }_{n+1} C_{i} 20^{i}(-64)^{n+1-i} g_{e}\left(\frac{x}{2^{2 n+2-i}}\right)
\end{aligned}
$$

for all $n \in \mathbb{N}_{0}$ and $x \in V$, which we get from (29) when we replace $n$ by $n+1$. 
Let $g, h \in S$ and let $K \in[0, \infty]$ be an arbitrary constant with $d(g, h) \leq K$. From the definition of $d$, we have

$$
\begin{aligned}
& \|J g(x)-J h(x)\| \leq 15\left\|g\left(\frac{x}{2}\right)-h\left(\frac{x}{2}\right)\right\| \\
& +5\left\|g\left(\frac{-x}{2}\right)-h\left(\frac{-x}{2}\right)\right\| \\
& +40\left\|g\left(\frac{x}{4}\right)-h\left(\frac{x}{4}\right)\right\| \\
& +24\left\|g\left(\frac{-x}{4}\right)-h\left(\frac{-x}{4}\right)\right\| \\
& \leq 64 K \Phi\left(\frac{x}{4}\right)+20 K \Phi\left(\frac{x}{2}\right) \\
& \leq L^{2} \frac{2(\sqrt{41}-5)^{2}}{32} K \Phi(x) \\
& +20 \frac{\sqrt{41}-5}{32} L K \Phi(x) \\
& \leq L K \Phi(x)
\end{aligned}
$$

for all $x \in V$, which implies that

$$
d(J g, J h) \leq L d(g, h)
$$

for any $g, h \in S$. That is, $J$ is a strictly contractive self-mapping of $S$ with the Lipschitz constant $L$.

Moreover, by (11), we see that

$$
\begin{aligned}
& \|f(x)-J f(x)\|=\left\|D f\left(\frac{x}{2}, \frac{x}{4}\right)+4 D f\left(\frac{x}{4}, \frac{x}{4}\right)\right\| \\
& \leq \varphi\left(\frac{x}{2}, \frac{x}{4}\right)+4 \varphi\left(\frac{x}{4}, \frac{x}{4}\right) \\
& \leq \frac{(\sqrt{41}-5)^{2} L^{2}}{32^{2}}(\varphi(2 x, x)+4 \varphi(x, x)) \\
& \quad \leq \frac{(\sqrt{41}-5)^{2} L^{2}}{32^{2}} \Phi(x)
\end{aligned}
$$

for all $x \in V$, which implies that

$$
d(f, J f) \leq \frac{(\sqrt{41}-5)^{2} L^{2}}{32^{2}}<\infty
$$

by the definition of $d$.

Therefore, according to Theorem 1 , the sequence $\left\{J^{n} f\right\}$ converges to the unique fixed point $F: V \rightarrow Y$ of $J$ in the set $T=\{g \in S: d(f, g)<\infty\}$, which is represented by (27) for all $x \in V$. Notice that

$$
d(f, F) \leq \frac{1}{1-L} d(f, J f) \leq \frac{(\sqrt{41}-5)^{2} L^{2}}{32^{2}(1-L)},
$$

which implies (26).
By definition of $F$, together with (11) and (25), we have

$$
\begin{aligned}
& \|D F(x, y)\|=\lim _{n \rightarrow \infty}\left\|D J^{n} f(x, y)\right\| \\
& =\lim _{n \rightarrow \infty} \| \sum_{i=0}^{n}{ }_{n} C_{i} 10^{i}(-16)^{n-i} f_{o}\left(\frac{x}{2^{2 n-i}}, \frac{y}{2^{2 n-i}}\right) \\
& +\sum_{i=0}^{n}{ }_{n} C_{i} 20^{i}(-64)^{n-i} f_{e}\left(\frac{x}{2^{2 n-i}}, \frac{y}{2^{2 n-i}}\right) \| \\
& \leq \lim _{n \rightarrow \infty} \sum_{i=0}^{n} \frac{{ }_{n} C_{i}}{2}\left(10^{i} 16^{n-i}+20^{i} 64^{n-i}\right) \\
& \times\left(\varphi\left(\frac{x}{2^{2 n-i}}, \frac{y}{2^{2 n-i}}\right)+\varphi\left(\frac{-x}{2^{2 n-i}}, \frac{-y}{2^{2 n-i}}\right)\right) \\
& \leq \lim _{n \rightarrow \infty} \sum_{i=0}^{n}{ }_{n} C_{i} 20^{i} 64^{n-i} \frac{(\sqrt{41}-5)^{n-i} L^{n-i}}{32^{n-i}} \\
& \times\left(\varphi\left(\frac{x}{2^{n}}, \frac{y}{2^{n}}\right)+\varphi\left(\frac{-x}{2^{n}}, \frac{-y}{2^{n}}\right)\right) \\
& \leq \lim _{n \rightarrow \infty} \sum_{i=0}^{n}{ }_{n} C_{i} 20^{i}(2(\sqrt{41}-5)){ }^{n-i}\left(\varphi\left(\frac{x}{2^{n}}, \frac{y}{2^{\mathrm{n}}}\right)\right. \\
& \left.+\varphi\left(\frac{-x}{2^{n}}, \frac{-y}{2^{n}}\right)\right) \leq \lim _{n \rightarrow \infty}(20+2(\sqrt{41}-5))^{n} \\
& \cdot\left(\varphi\left(\frac{x}{2^{n}}, \frac{y}{2^{n}}\right)+\varphi\left(\frac{-x}{2^{n}}, \frac{-y}{2^{n}}\right)\right) \leq \lim _{n \rightarrow \infty} 2^{n}(\sqrt{41} \\
& +5)^{n} \frac{(\sqrt{41}-5)^{n} L^{n}}{32^{n}}(\varphi(x, y)+\varphi(-x,-y)) \\
& \leq \lim _{n \rightarrow \infty} L^{n}(\varphi(x, y)+\varphi(-x,-y))=0
\end{aligned}
$$

for all $x, y \in V$; that is, $F$ is a solution of the functional equation (1). Notice that if $F$ is a solution of the functional equation (1), then the equality

$$
F(x)-J F(x)=D F\left(\frac{x}{2}, \frac{x}{4}\right)+4 D F\left(\frac{x}{4}, \frac{x}{4}\right)
$$

implies that $F$ is a fixed point of $J$.

\section{Competing Interests}

The authors declare that there is no conflict of interests regarding the publication of this paper.

\section{Authors' Contributions}

All authors contributed equally to the writing of this paper. All authors read and approved the final paper.

\section{Acknowledgments}

Soon-Mo Jung was supported by Basic Science Research Program through the National Research Foundation of Korea (NRF) funded by the Ministry of Education (no. 2013R1A1A2005557). 


\section{References}

[1] S. M. Ulam, A Collection of Mathematical Problems, Interscience, New York, NY, USA, 1960.

[2] D. H. Hyers, "On the stability of the linear functional equation," Proceedings of the National Academy of Sciences of the United States of America, vol. 27, pp. 222-224, 1941.

[3] P. Găvruta, "A generalization of the Hyers-Ulam-Rassias stability of approximately additive mappings," Journal of Mathematical Analysis and Applications, vol. 184, no. 3, pp. 431-436, 1994.

[4] T. M. Rassias, "On the stability of the linear mapping in Banach spaces," Proceedings of the American Mathematical Society, vol. 72, no. 2, pp. 297-300, 1978.

[5] J. R. Lee, J.-h. Kim, and C. Park, "A fixed point approach to the stability of an additive-quadratic-cubic-quartic functional equation," Fixed Point Theory and Applications, vol. 2010, Article ID 185780, 16 pages, 2010.

[6] L. Cădariu and V. Radu, "Fixed points and the stability of Jensen's functional equation," Journal of Inequalities in Pure and Applied Mathematics, vol. 4, no. 1, article 4, 2003.

[7] L. Cădariu and V. Radu, "Fixed points and the stability of quadratic functional equations," Analele Universitatii din Timisoara, Seria Matematică-Informatică, vol. 41, pp. 25-48, 2003.

[8] L. Cădariu and V. Radu, "On the stability of the Cauchy functional equation: a fixed point approach," in Iteration Theory, vol. 346 of Grazer Mathematische Berichte, pp. 43-52, KarlFranzens-Universitäet, Graz, Graz, Austria, 2004.

[9] J. B. Diaz and B. Margolis, "A fixed point theorem of the alternative, for contractions on a generalized complete metric space," Bulletin of the American Mathematical Society, vol. 74, pp. 305-309, 1968.

[10] Y.-H. Lee, "On the generalized Hyers-Ulam stability of the generalized polynomial function of degree 3," Tamsui Oxford Journal of Mathematical Sciences, vol. 24, no. 4, pp. 429-444, 2008.

[11] S.-M. Jung, "A fixed point approach to the stability of a Volterra integral equation," Fixed Point Theory and Applications, vol. 2007, Article ID 57064, 9 pages, 2007.

[12] S.-M. Jung and T.-S. Kim, "A fixed point approach to the stability of the cubic functional equation," Boletín de la Sociedad Matemática Mexicana, vol. 12, no. 1, pp. 51-57, 2006. 


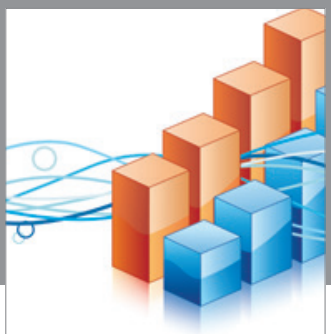

Advances in

Operations Research

vatem alat4

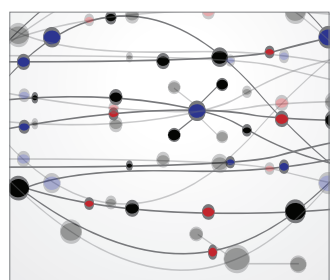

\section{The Scientific} World Journal
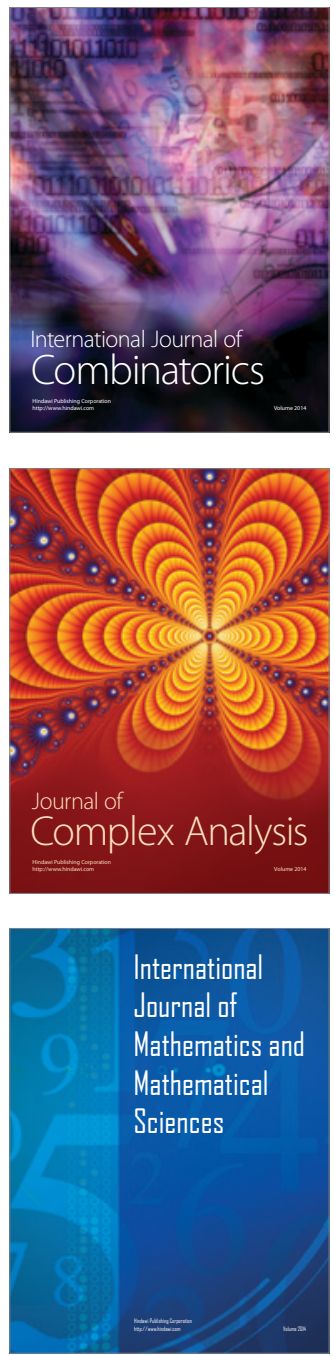
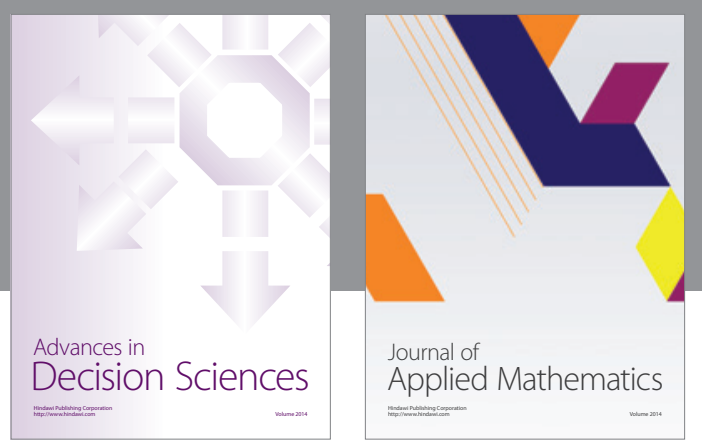

Algebra

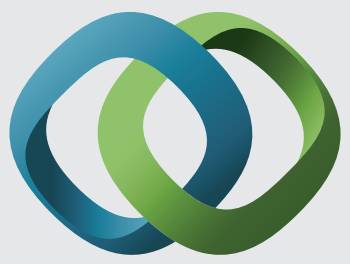

\section{Hindawi}

Submit your manuscripts at

http://www.hindawi.com
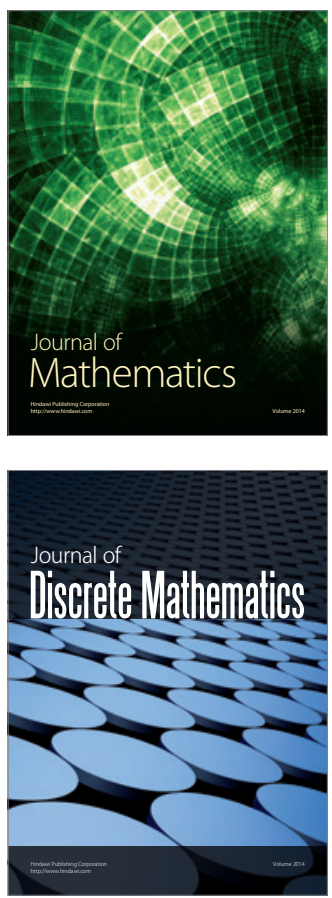

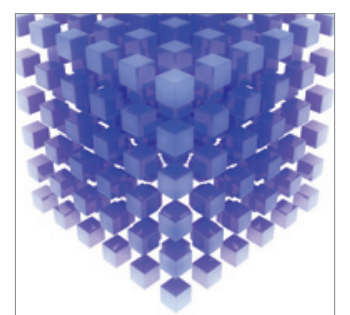

Mathematical Problems in Engineering
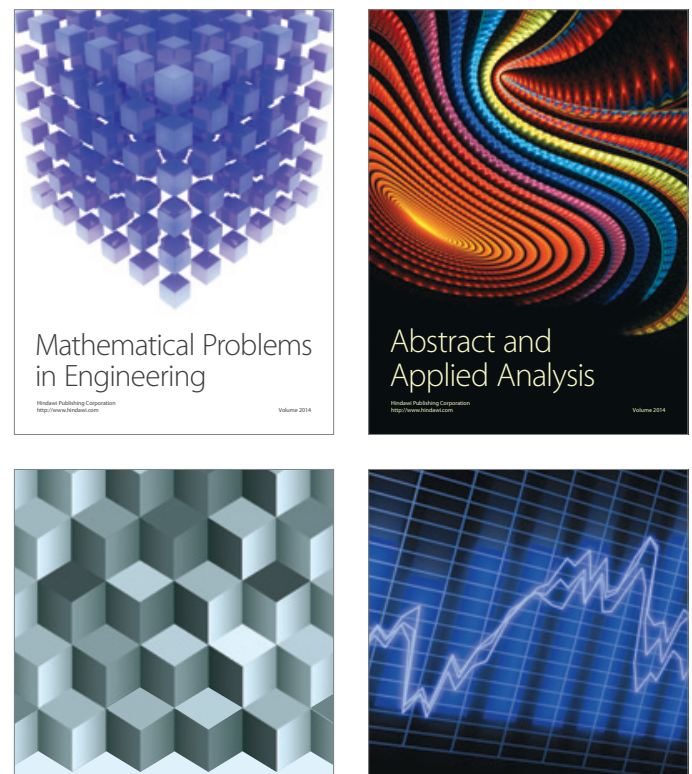

Journal of

Function Spaces

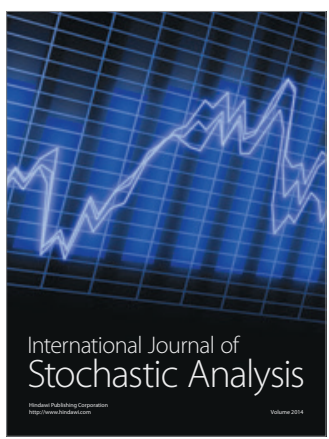

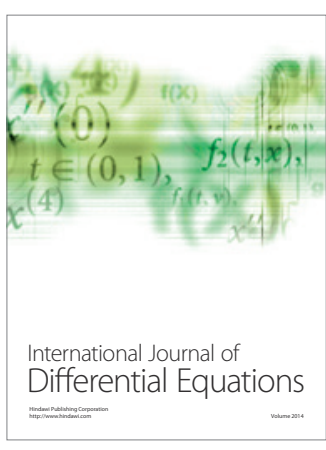
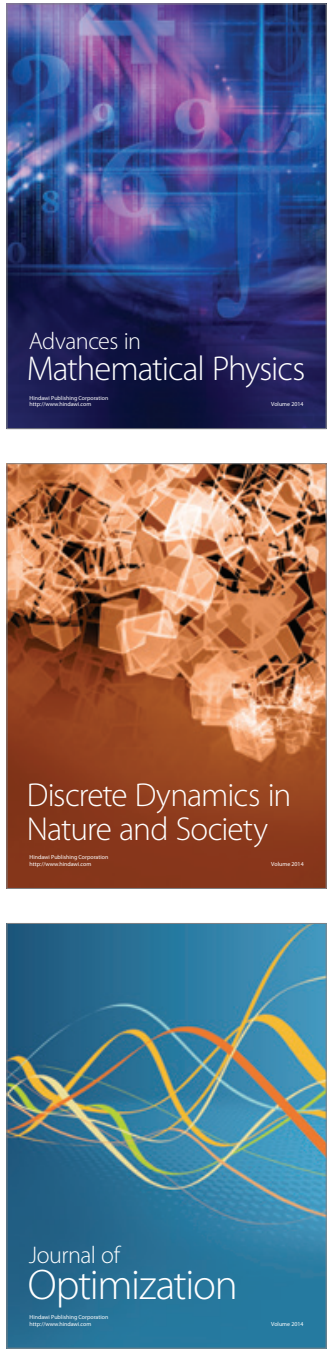